\title{
Tumor Placentário Diagnosticado Durante a Gravidez: Relato de Caso
}

\author{
Placental Tumor Diagnosed in Pregnancy: A Case Report
}

Francisco Mauad Filho, Antonio Gadelha da Costa, Patricia Spara, Adilson Cunha Ferreira Reginaldo Antônio de Oliveira Freitas Júnior, Maria Matheus de Sala, Fábio Valiengo Valeri

\section{RESUMO}

\begin{abstract}
O tumor não trofoblástico placentário encontrado com maior freqüência é o corioangioma, com incidência de aproximadamente $1 \%$. Quando são pequenos, geralmente não levam a alterações fetais, mas quando são grandes, podem levar a restrição de crescimento intra-útero, poliidrâmnio, trabalho de parto prematuro, insuficiência cardiaca congestiva e morte fetal. Os autores relatam um caso de corioangioma em uma paciente de 28 anos, diagnosticado em exame ultrasonográfico de rotina, com idade gestacional de 32 semanas. O diagnóstico foi confirmado pelo exame anatomopatológico. As avaliações ultra-sonográficas revelaram a presença de sofrimento fetal crônico, que levou à interrupção da gestação com 36 semanas. Os resultados neonatais foram satisfatórios, com Apgar de 9-10 e peso fetal de 2.460 gramas.
\end{abstract}

PALAVRAS-CHAVE: Placenta. Corioangioma. Dopplervelocimetria. Complicações da gravidez. Crescimento intra-uterino restrito.

\section{Introdução}

Os conhecimentos sobre placentação e transferência de nutrientes, gases e metabólitos da mãe para o feto contribuíram para que a placenta fosse considerada órgão de especial importância nas gestações normais e de alto risco, como também no metabolismo e crescimento fetal. Neste contexto, estão incluídas as mudanças fisiológicas que ocorrem nas artérias espiraladas durante o processo de invasão trofoblástica ${ }^{1}$, as publicações sobre a participação do hCG $\beta$ na placentação pela liberação de citocinas $^{2}$ e as alterações encontradas nos receptores placentários da renina angiotensina aldosterona em pacientes com préeclâmpsia e crescimento intra-uterino restrito, fatores estes responsáveis pela diminuição do fluxo sangüíneo útero-placentário ${ }^{3}$. Outras patologias que podem interferir nos mecanismos de transporte útero-placentário e no aporte sangüineo para o concepto são a doença hemolítica perinatal, a sindrome antifosfolipide, as doenças infecciosas e os tumores placentários. Nestes últimos inserem-

Departamento de Ginecologia e Obstetrícia da Faculdade de Medicina de Ribeirão Preto da Universidade de São Paulo Correspondência: Francisco Mauad Filho

Av. dos Bandeirantes, $3.900-8^{\circ}$ andar - Campus Universitário

14049-900 - Ribeirão Preto - São Paulo

Fone: (16) 602-2587

e-mail: $\underline{\text { mauad@ultra-sonografia.com.br }}$ se os tumores trofoblásticos, os angiomas e os teratomas.

Os angiomas, frequëntemente designados corioangiomas ou corangiomas, são tumores placentários formados por capilares ou sinusóides de dimensões variadas, únicos ou múltiplos, que se localizam com maior freqüência na placa corial, projetando-se para a cavidade amniótica ${ }^{4}$. Usualmente quando são pequenos não levam a alterações maternas e fetais importantes, porém, geralmente, quando são grandes, comprometem a função placentária, podendo levar a restrição de crescimento intra-uterino, malformação fetal, hidropisia fetal não imune e parto pré-termo ${ }^{5}$. Entretanto, também se tem verificado a descrição da associação do coriangioma com poliidrâmnio ${ }^{5}$, inclusive com resultado pós-natal de feto normal ${ }^{6}$.

A ultra-sonografia permite a detecção precoce de tumores placentários, de forma que este método tem sido largamente empregado no diagnóstico do corioangioma, com beneficios indiscutíveis no prognóstico materno-fetal.

Por sua vez, a dopplervelocimetria pode exercer um papel no diagnóstico diferencial entre corioangioma e outros tumores placentárias como hematomas, teratomas e molas parciais, por apresentar, em se tratando de corioangioma, aumento da vascularização no interior do tumor ${ }^{7}$.

A detecção precoce do corioangioma é de grande importância no prognóstico materno-fetal, 
porque desempenha significante aumento no risco de parto pré-termo e morte fetal.

A ablação vascular intra-útero por via endoscópica tem sido utilizada nos corioangiomas de grandes dimensões, com o objetivo de suprimir o fluxo sangüíneo para o tumor, responsável pela formação de fistulas artério-venosas e tromboses, que podem culminar em alterações fetais graves, como anemia, hidropisia e parada cardiaca ${ }^{8}$.

\section{Relato do Caso}

Paciente A.M.F.B.K., 28 anos, gesta 2 para 1, cesariana por apresentação pélvica em 1995, com peso fetal de $3.470 \mathrm{~g}$. A primeira consulta prénatal foi realizada no dia 17 de julho de $2001 \mathrm{com}$ idade gestacional calculada pela data da última menstruação de 6,4 semanas e idade gestacional ecográfica de 5 semanas. A paciente apresentava-se assintomática. Durante a evolução da gravidez não observamos anormalidades no exame clínico. Os níveis tensionais, pulso, altura do fundo uterino, circunferência abdominal e batimento cardiaco fetal foram normais. Não apresentou edema e aumento de peso foi de $12 \mathrm{~kg}$ durante toda a gestação.

Os exames complementares da primeira consulta pré-natal mostraram hemoglobina de $11,4 \mathrm{~g} / \mathrm{dL}$, hematócrito de $34 \mathrm{~g} \%$ e leucócitos de $6.100 / \mathrm{mm}^{3} \mu \mathrm{L}$. A glicemia foi de $73 \mathrm{mg} / \mathrm{dL}$ e a urina I e colpocitologia oncótica apresentaram-se normais. As provas sorológicas não mostraram resultados significativos para a gestação atual.

Em exame ultra-sonográfico na $25^{\text {a }}$ semana, observou-se que o feto apresentava dolicocefalia, com índice cefálico de $66 \%$. Na oportunidade, a relação fêmur/ circunferência abdominal era de $22,3 \%$ e o peso fetal $858 \mathrm{~g}$. A partir desta observação foi investigada a presença de outra alteração ultra-sonográfica que pudesse estar associada à dolicocefalia. A ultra-sonografia de 23 de janeiro de 2002 (correspondendo à idade gestacional de 32 semanas) mostrou na placa corial, com protusão para a cavidade amniótica, uma imagem hipoecóica, medindo $7,5 \times 6,2 \times 6,1 \mathrm{~cm}$, com volume de $150 \mathrm{~cm}^{3}$. À avaliação pela dopplervelocimetria notava-se rica vascularização (Figura 1). As avaliações ultra-sonográficas com Doppler e os resultados do índice de líquido amniótico (ILA) estão apresentados nas Tabelas 1 e 2 .

No dia 23 de fevereiro de 2002, com idade gestacional de 36,2 semanas, a paciente foi submetida à cesariana, cujas indicações foram: sofrimento fetal crônico, caracterizado pela oligoidramnia e à dopplervelocimetria, apresentação pélvica e tumor placentário. O índice de Apgar foi 9 e 10 no primeiro e quinto minutos, respectivamente. O peso fetal foi de $2.460 \mathrm{~g}$. A placenta, junto com a tumoração e o cordão umbilical, foi enviada para estudo anatamopatológico.

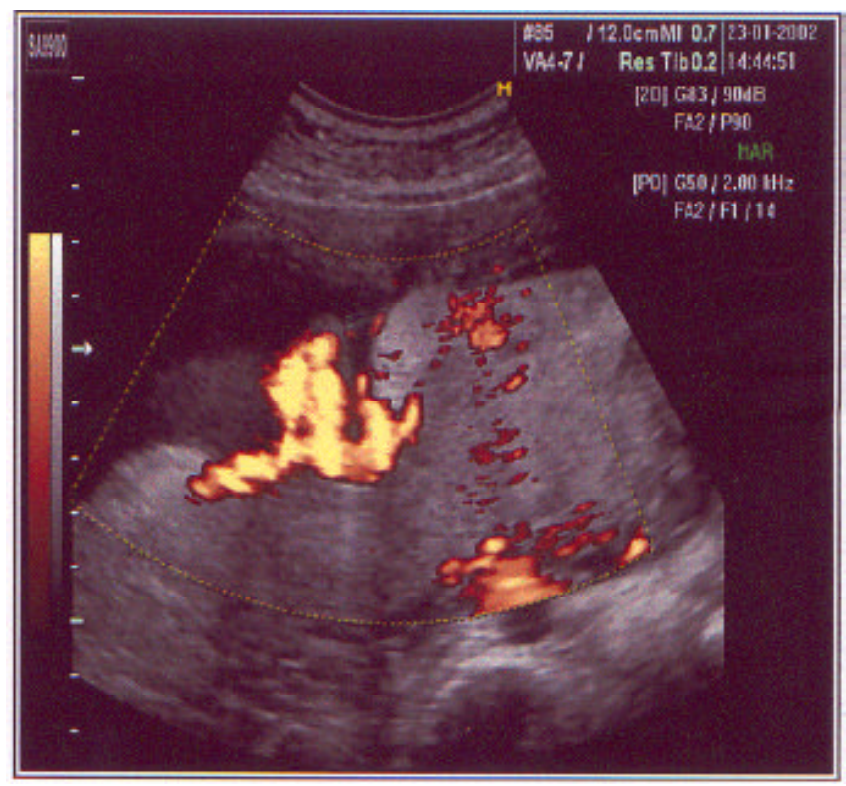

Figura 1 - Tumor placentário ao ultra-som com Doppler. Observar a presença do tumor localizado ao nível da placa corial com protusão para a cavidade amniótica, e a hipervascularização ao Doppler.

Tabela 1 - Evolução dos exames ultra-sonográficos.

\begin{tabular}{cccccc}
\hline Data (US) & IG (UM) & IG (US) & F/CA & PF (g) & Tumor $\left(\mathbf{c m}^{3}\right)$ \\
\hline $5 / 12 / 01$ & 26,5 & 25 & 22,3 & 858 & - \\
$23 / 01 / 02$ & 33,4 & 32 & 22,7 & 1769 & 159 \\
$06 / 02 / 02$ & 35,5 & 34 & 22,5 & 2172 & 182 \\
$14 / 02 / 02$ & 36,6 & 35,1 & & & 217 \\
$20 / 02 / 02$ & 37,5 & 36 & 23,6 & 2376 & 234 \\
$22 / 02 / 02$ & 38,1 & 36,2 & 23,6 & 2502 & 331 \\
\hline
\end{tabular}

US: ultra-sonografia; IG: idade gestacional; UM: última menstruação; F: fêmur; CA: circunferência abdominal; PF: peso fetal.

Tabela 2 - Evolução dos exames ultra-sonográficos com dopplervelocimetria e valores do ILA.

\begin{tabular}{lcccc}
\hline Data & IG (US) & AU & ACM & ILA (cm) \\
\hline $5 / 12 / 01$ & 25 & & & 11 \\
$23 / 01 / 02$ & 32 & IR: 58 & IR: 74 & 13 \\
$06 / 02 / 02$ & 34 & IR: 50 & IR: 86 & 12 \\
14/02/02 & 35,1 & IR: 58 & IR: 74 & 13 \\
20/02/02 & 36 & IR: 70 & IR: 70 & 10 \\
22/02/02 & 36,2 & IR: 70 & IR: 68 & 3,4 \\
\hline
\end{tabular}

IG: idade gestacional; US: ultra-sonografia; AU: artéria umbilical; ACM: artéria cerebral média; ILA: índice de líquido amniótico; IR: índice de residência. 
$\mathrm{Na}$ macroscopia a placenta apresentava forma discóide, com peso de $710 \mathrm{~g}$ e medindo $20,0 \mathrm{x}$ $16,0 \times 4,0 \mathrm{~cm}$ (volume de $665 \mathrm{~cm}^{3}$ ), cor pardo-acastanhada e cinza-azulada na face fetal, cotilédones mal definidos, placa corial com vasos calibrosos e superficie de corte congesta. Observava-se um nódulo hemorrágico, vinhoso, circunscrito, algo friável, distinto do tecido placentar circunjacente, de forma ovalada, medindo $6,5 \times 6,0 \times 5,5 \mathrm{~cm}$ nos maiores diâmetros, perfazendo um volume de aproximadamente $111 \mathrm{~cm}^{3}$, o que corresponde a cerca de $16 \%$ do volume placentário. Foram observados vasos ectásicos nas proximidades da implantação funicular, zonas focais de infarto, calcificações esparsas e delicadas. As membranas encontravam-se translúcidas e o cordão umbilical media $20 \mathrm{~cm}$ de comprimento, espessura de $1,0 \mathrm{~cm}$, com duas artérias e uma veia (Figura 2).

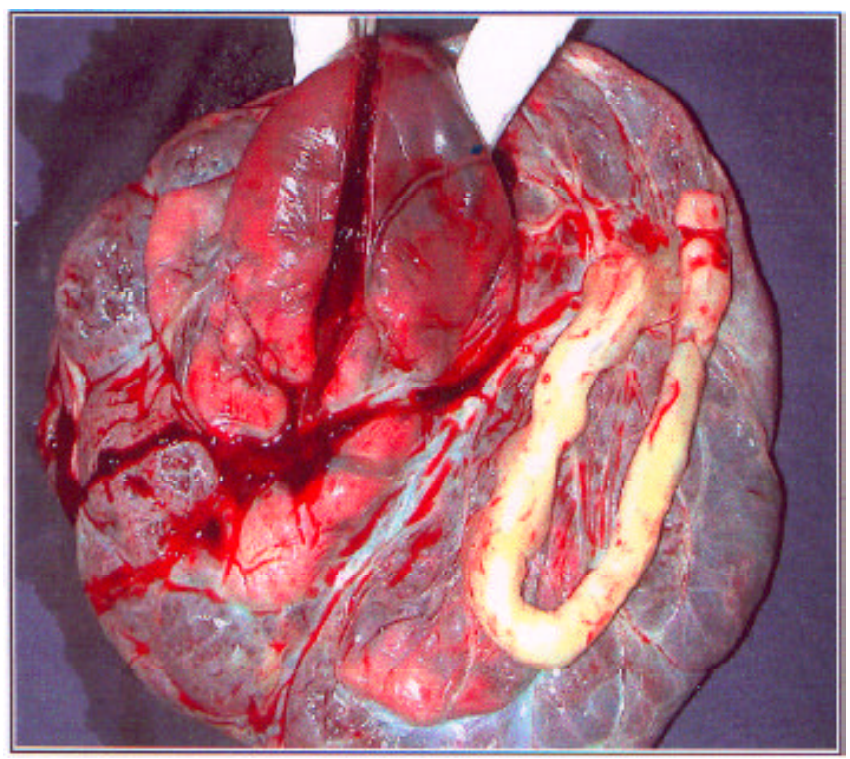

Figura 2 - Aspecto macroscópico do corioangioma. Notar a presença do nódulo hemorrágico, vinhoso, circunscrito, distinto do tecido placentar circunjacente, como também vasos ectásicos próximo à implantação funicular.

Ao exame microscópico, a placa basal e a corial encontravam-se edemaciadas, com infartos isquêmicos e vasos congestos. As vilosidades coriônicas mostravam-se pequenas, unilaminares, com hipervascularização e microcalcificações, algumas com nós sinciciais e formação de membrana sincício-vascular. Os citotrofoblastos apresentavam distribuição habitual e os nós sinciciais eram freqüentes, com microcalcificações. Observava-se calcificação focal e vascularização congesta e bem desenvolvida. Não foram visualizados agentes microbiológicos, nem infiltrado inflamatório. As membranas estavam edemaciadas, isquêmicas e com reação flogística focal. O cordão apresentava-se edemaciado, com padrão pseudomixóide na parede, junto à geléia de Wharton, na sua base de implantação. A massa vinhosa era composta de material hemorrágico e necrótico, identificando-se focalmente esboço de arquitetura vascular capilar, por vezes anastomosada, na forma de lagos venosos e sem atipias citonucleares endoteliais.

O diagnóstico histopatológico foi de placenta no terceiro trimestre gestacional, compativel com 34-35 semanas, insuficiência placentária, membranite inespecífica focal, cordão edemaciado com padrão pseudomixóide e corioangioma de padrão hemangiomatoso hemorrágico.

\section{Discussão}

Os tumores que podem ser encontrados na placenta podem ser de origem extrinseca ou de origem placentária. Os tumores extrínsecos são raros e provenientes de metástase, principalmente por infiltrados leucêmicos. Os tumores de origem placentária podem originar-se do trofoblasto, o que constitui a doença trofoblástica gestacional, variedade mais freqüente de tumores placentários, e os tumores do estroma, onde estão incluídos os teratomas e o corioangioma ${ }^{9}$.

Há mais de dois séculos tem-se relatado sobre o corioangioma placentário, porém com uma incidência extremamente conflitante ${ }^{10}$. Entretanto, acredita-se que o corioangioma placentário incide em cerca de $1 \%$ das placentas ${ }^{11}$.

A aquisição de metodologias especializadas na avaliação materno-fetal realizada durante o pré-natal, dentre elas a ultra-sonografia bidimensional, a dopplervelocimetria e mais recentemente a ultra-sonografia tridimensional, possibilitou a detecção eventual de tumores placentários, antes diagnosticados apenas no período pós-natal. A aparência ultra-sonográfica do corioangioma é de uma massa bem circunscrita, predominantemente hipoecogênica, localizada freqüentemente próximo à superficie coriônica e à inserção do cordão umbilical ${ }^{12}$.

$\mathrm{O}$ caso em estudo mostrou características ultra-sonográficas de tumor hiperecogênico, localizado na placa corial, com protusão para a cavidade amniótica e com rica vascularização na dopplervelocimetria, o que nos permitiu diagnosticálo diferencialmente de hemorragia subcorial ou intervilosa ${ }^{9}$. Ainda mais, a presença de vascularização no tumor pode ser um fator relevante no prognóstico da gestação, pois os tumores placentários que contêm numerosos lagos venosos podem estar relacionados a polidrâmnio e insuficiência cardíaca fetal ${ }^{5}$. 
Geralmente, os corioangiomas são tumores que apresentam evolução benigna e seu tamanho está diretamente relacionado às repercussões materno-fetais, de forma que, quando são grandes, podem portar-se como fistulas artério-venosas, levando a restrição de crescimento intra-útero, insuficiência cardiaca fetal com poliidrâmnio e hidropisia, por vezes terminando em parto prematuro e morte fetal ${ }^{13}$. Os corioangiomas, quando maiores do que $4 \mathrm{~cm}$ de diâmetro, associam-se a perda fetal de aproximadamente $40 \%{ }^{8}$.

Por outro lado, tumores pequenos podem não estar relacionados a comprometimento maternofetal, de modo que muitas vezes não há complicações para a mãe ou para o feto, quando o tumor apresenta pequenas dimensões, até cerca de 6 centimetros.

Entretanto, os corioangiomas de pequenas dimensões, no início da gravidez, podem crescer ao longo da gestação, levando a alterações importantes no leito placentário e, conseqüentemente, aumento no risco de morte fetal ${ }^{12}$.

Verificou-se no corioangioma em estudo associação com sofrimento fetal crônico caracterizado pela oligoidramnia e modificações na dopplervelocimetria, a saber: diminuição do índice de resistência na artéria cerebral média e aumento do índice de resistência na artéria umbilical. Outro achado encontrado foi a presença de dolicocefalia, cuja associação com o corioangioma não foi referida na literatura pesquisada.

Do ponto de vista histopatológico, o tumor descrito neste trabalho apresentou características tipicas de um corioangioma angiomatoso, que por sua característica funciona como verdadeira fistula artério-venosa, o que pode ter explicado a presença de insuficiência placentária. Ainda mais, como descrito por Benirschke e Kaufmann ${ }^{4}$, em 1995, a participação da geléia de Wharton nestes casos pode ter conferido o padrão mixomatoso observado.

Apesar de vários estudos feitos durante décadas, a placenta continua sendo um dos órgãos de dificil conhecimento fisiopatológico. Várias condições placentárias capazes de comprometer a evolução da gestação e o desenvolvimento fetal não eram passiveis de detecção durante o periodo prénatal. A ultra-sonografia, como método de excelência, trouxe novas possibilidades à obstetrícia, permitindo nova forma de acesso ao ambiente intrauterino, possibilitando o diagnóstico precoce de anomalias placentárias, antes detectadas apenas no periodo pós-parto pelos estudos anátomo-patológicos. Nisto inserem-se os corioangiomas, tumores placentários que pela sua própria morfologia variam desde tamanhos microscópicos até dimensões possiveis de serem diagnosticadas nos exames ultra-sonográficos de rotina.

Mesmo sabendo-se que os tumores placen- tários de grandes dimensões relacionam-se a prognósticos sombrios, os corioangiomas de pequenas dimensões também devem ser valorizados, pelo risco de parto pré-termo e morte fetal ${ }^{14}$. Desta forma, devemos salientar a importância no diagnóstico precoce do corioangioma como conduta relevante para a vigilância materno-fetal acurada e conseqüente redução no risco de perda conceptual.

Importante salientar que o diagnóstico ultrasonográfico do corioangioma em estudo permitiu a detecção precoce do mesmo, tendo nos conduzido a um acompanhamento adequado e ao diagnóstico do comprometimento fetal, contribuindo com a decisão na resolução da gestação e, conseqüentemente, evitando complicações graves ocasionadas pelos corioangiomas placentários, que terminam, na maioria das vezes, em óbito fetal.

Desta forma, concluímos enfatizando a importância dos exames ultra-sonográficos em fases precoces da gravidez, tendo em vista ser este método propedêutico extremamente importante na detecção precoce de doenças que possam comprometer a saúde materno-fetal. No que se refere ao corioangioma, este deve ter acompanhamento adequado, pois a evolução tumoral está diretamente relacionada ao comprometimento fetal, sendo este fator preponderante na tomada de condutas diante de gestantes portadoras de corioangioma placentário.

Finalmente, sugere-se que a placenta seja considerada órgão de grande importância desde a sua formação até o seu delivramento, merecendo atenção especial tanto nas gestações normais quanto nas de alto risco, contribuindo, deste modo, com a redução dos índices de mortalidade materna e fetal.

\section{ABSTRACT}

The most frequently nontrophoblastic tumor of the placenta found is chorioangioma, with an incidence of about $1 \%$. When they are small, they do not significantly affect the fetus, but the large ones can cause intrauterine growth restriction, polyhydramnios, premature delivery, congestive heart failure and fetal death. The authors report a case of chorioangioma in a 28-year-old woman, second gestation, whose diagnosis was established at the $32^{\text {nd }}$ week by ultrasound and confirmed by the anatomopathological examination. Ultrasonography evaluations showed chronic fetal distress and the delivery was performed at 36 weeks. The newborn results were satisfactory with Apgar 9-10 and fetal weight $2.460 \mathrm{~g}$.

KEY WORDS: Placenta. Chorioangioma. Doppler ultrasonography. Complication of pregnancy. Intrauterine growth restriction. 


\section{Referências}

1. Stone S, Khamashta MA, Poston L. Placentation, antiphospholipid syndrome and pregnancy outcome. Lupus 2001; 10:67-74.

2. Licht P, Russu V, Wildt L. On the role of human chorionic gonadotropin (hCG) in the embryoendometrial microenvironment: implications for differentiation and implantation. Semin Reprod Med 2001; 19:37-47.

3. Stoz F, Keckstein G, Schuhmann RA. Histometric case report of a placenta following intra-uterine therapy of rhesus incompatibility. Geburtshilfe Frauenheilkd 1987; 47:349-50.

4. Benirschke K, Kaufmann P. Benign tumors. In: Benirschke K, Kaufmann P, editors. Pathology of the Human Placenta. $3^{\text {rd }}$ ed. New York: SpringerVerlag; 1995. p.709.

5. Jauniaux E, Ogle R. Color Doppler imaging in the diagnosis and management of chorioangiomas. Ultrasound Obstet Gynecol 2000; 15:463-7.

6. Kriplani A, Abbi M, Banerjee N, Roy KK, Takkar D. Indomethacin therapy in the treatment of polyhydramnios due to placental chorioangioma. J Obstet Gynaecol Res 2001; 27:245-8.

7. Prapas N, Liang RI, Hunter D, et al. Color Doppler imaging of placental masses: differential diagnosis and fetal outcome. Ultrasound Obstet Gynecol 2000; 16:559-63.
8. Quintero RA, Reich H, Romero R, Johnson MP, Gonçalves L, Evans MI. In utero endoscopic desvascularization of a large chorioangioma. Ultrasound Obstet Gynecol 1996; 8:48-52.

9. Zanforlin Filho SM. Placenta. In: Pastore AR, Cerri GG, editors. Ultra-Sonografia Obstetrícia Ginecologia. $1^{\text {a }}$ ed. São Paulo: Sarvier; 2000. p.155.

10. Fox H. Vascular tumors of the placenta. Obstet Gynecol Surv 1967; 22:697-711.

11. Mancuso A, D’Anna R, Corrado F, Cannata ML. Large placental chorioangioma. Acta Obstet Gynecol Scand 2001; 80:965-6.

12. Batukan C, Holzgreve W, Danzer E, Bruder E, Hösli I, Tercanli S. Large placental chorioangioma as a cause of sudden intrauterine fetal death. A case report. Fetal Diagn Ther 2001; 16:394-7.

13. Matheus M, Barbieri Neto J, Tedesco JJA, Sala MA, Franzolin A, Barrionovo N. Corioangioma: estúdio clínico y anátomo-patológico de ocho casos. Clin Invest Ginecol Obstet 1976; 3:327-33.

14. Bashiri A, Furman B, Erez O, Wiznitzer A, Holcberg G, Mazor M. Twelve cases of placental chorioangioma. Pregnancy outcome and clinical significance. Arch Gynecol Obstet 2002; 266:53-5.

Recebido em: 20/5/2002 Aceito com modificações em: 8/8/2002

\title{
COMUNICADO AOS ASSOCIADOS E LEITORES
}

\section{FEBRASGO na Internet}

Para maiores informações temos à disposição quatro endereços eletrônicos:

\author{
febrasgopresiden@uol.com.br \\ secretaria.executiva@febrasgo.org.br \\ publicacoes@febrasgo.org.br \\ tego.habilitacoes@febrasgo.org.br
}

